\section{Psychosis, epileptiform abnormalities and clozapine}

SIR: The atypical antipsychotic clozapine was reintroduced for the treatment of resistant schizophrenia in January 1990 . Approximately $20 \%$ of schizophrenic patients are resistant to typical antipsychotic medication (Davis et al, 1980) and of those who do respond initially, approximately $20-30 \%$ are liable to relapse despite maintenance therapy (Kane \& Lieberman, 1987). A small but significant number of resistant cases have been suggested as having an organic cause, although the role of the organic factors remains unclear (Kolokowska et al, 1985). One such factor to be considered is cryptic epileptiform conditions. In routine clinical practice clozapine is often immediately substituted or dove-tailed into pre-existing medication. In the patient with an organic schizophreniform psychosis this may not only mitigate against a response to clozapine, but may actually worsen the condition. The following case illustrates this.

Case report. A 20-year-old single man was referred for tertiary opinion with a 2.5 year history of (resistant) schizophreniform symptoms. Initial presentation in November 1988 followed a two-day history of psychotic symptoms, believing he was Jesus Christ, and becoming increasingly socially withdrawn. The only past history of note was a two-month history of increasing cannabis use.

Symptoms failed to subside and he was started on neuroleptics. Over the subsequent 2.5 years, the course of illness was characterised by episodic delusions of being Jesus Christ with increasing psychotic features and agitation. These lasted between 4 and 10 days with a $2-4$ day period of resolution. Therapeutic interventions included various typical neuroleptics (oral and depot), lithium, antidepressants, and electroconvulsive therapy, with no effect. Clozapine, up to $1100 \mathrm{mg}$, failed to ameliorate his symptoms. An electroencephalogram (EEG) recorded near the beginning of the illness revealed frequent short bursts of high voltage activity, generalised in character, and excess slow activity in the left hemisphere - reported as abnormal with epileptiform equivalents. The computerisedtomography scan was normal. At the time of referral, the EEG while on clozapine and benzodiazepines continued to show non-specific severe diffuse slowing of background rhythms. He was slowly withdrawn from all medication. Subsequent EEG on no medication showed a significant improvement with mild diffuse slowing and occasional general slowing and nonspecific slow waves.

He was subsequently started on carbamazepine, increasing to $400 \mathrm{mg}$ b.d. At this time his behaviour was characterised by longer periods of resolution and shorter less severe paroxysmal relapses. Unfortunately his compliance became erratic with eventual refusal. However, unmedicated he felt much improved and while maintaining some of his beliefs, he did not feel these excluded him from a normal lifestyle. $\mathrm{He}$ was therefore referred back for rehabilitation and community placement.
This patient probably has an ictal psychosis. This in itself is of interest as these are relatively unusual compared with inter-ictal psychoses. Generalised seizures (Haller \& Birder, 1990) and epileptiform EEG abnormalities (Schmauss et al, 1989) have been reported on clozapine. His psychosis was apparently exacerbated by the proconvulsant action of clozapine, and his clinical and EEG improvement following withdrawal of clozapine illustrate this fact. Thus we propose that before assigning a diagnosis of treatment-resistant schizophrenia, all antipsychotics should be stopped and patients investigated for an alternative explanation for drug-resistant symptoms. Clozapine should also be used cautiously where epileptiform mechanisms may be operating in the context of a psychotic illness.

We would like to thank Dr M. Launer of Burnley General Hospital for allowing us to report this case.

DA VIS, J. M., SChAFFER, C. B., KILliAN, G. A., et al (1980) Important issues in the drug treatment of schizophrenia. Schizophrenia Bulletin, 6, 70-87.

Haller, E. \& Birder, R. I. (1990) Clozapine and seizures. American Journal of Psychiatry, 147, 1069-1071.

KANE, J. M. \& LIEBERMAN, J. A. (1987) Maintenance pharmacotherapy in schizophrenia. In Psychopharmacology, the Third Generation of Progress; the Emergence of Molecular Biology and Biological Psychiatry (ed. H. Y. Meltzer), pp. 1103-1109. New York: Raven Press.

Kolokowska, T., Williams, A. O., Ardern, M., et al (1985) Schizophrenia with good and poor outcome. British Journal of Psychiatry, 146, 229-246.

Schmauss, M., Wolff, R., ERfurth, A., et al (1989) Tolerability of long term clozapine treatment. Psychopharmacology. 99, 5105-5108.

J. MUNRO

Maudsley Hospital

N. GODDARD

Denmark Hill

London SES

\section{Catatonia and creatinine phosphokinase}

SIR: I would like to report the recurrent association between catatonia and raised creatinine phosphokinase (CPK) in a schizophrenic patient.

The recent resurgence of interest in catatonia is mainly due to the fact that catatonic symptoms are often part of the neuroleptic malignant syndrome (NMS). This has raised the question whether catatonia could be a risk factor in developing NMS (White \& Robins, 1991). The difficulty of differentiating the two conditions clinically underlines the need for laboratory tests. High white blood count, raised CPK, and recently low serum iron have all been associated with NMS (Rosebush \& Mazurek, 1991). 
Case report. Mr A is 47-years old and has suffered from schizophrenia since the age of 24 . His illness had followed a favourable course with maintenance neuroleptic. He was admitted to hospital for the first time in 1990 in a psychotic state with persecutory delusions, thought disorder, and behaviour disturbance. On the fourth day of his admission after having received $200 \mathrm{mg}$ of oral chlorpromazine (CPZ) daily he developed catatonic features (eg. standing to attention unresponsive, posturing, and showing intermittent "muscle stiffness"). The blood results on that day showed no abnormality besides raised CPK at $1331 \mathrm{U} / 1$ (normal range is $0-175 \mathrm{U} / 1$ ). He had not had any trauma nor did he have any evidence of liver or cardiac pathology. Lorazepam was substituted for CPZ and a week later the catatonic signs had resolved and the CPK was $71 \mathrm{U} / 1$ Unfortunately his psychotic symptoms required the resumption of maintenance therapy with thioridazine.

He was readmitted 14 months later in an acute catatonic state and on admission the CPK was raised at $3550 \mathrm{U} / \mathrm{l}$. He had stopped thioridazine five days previously on his own initiative. Initially he was agitated and his restless pacing was interrupted abruptly by long periods of immobility and staring. He would not address people spontaneously but when interviewed showed loosening of association and echolalia. After a few days he became stuporous and mute, he took to his bed refusing to eat or drink except from a relative. At times he showed "waxy flexibility" and at others active resistance to being moved. After two weeks of treatment with lorazepam his catatonic symptoms improved and the CPK normalised $(84 \mathrm{U} / \mathrm{l})$ but he remained mute and very withdrawn. Thioridazine $(600 \mathrm{mg} /$ day $)$ was substituted for lorazepam with a short-lived improvement. He recovered after six treatments with ECT in addition to thioridazine on which he was discharged again. The CPK after ECT was $47 \mathrm{U} / 1$.

Schizophrenic patients represent only a fraction of all catatonic patients (Fein \& McGrath, 1990) but their need for long-term neuroleptic treatment puts them at risk of developing NMS. The rise and fall of CPK seem to mirror the intensity of the catatonic symptoms in both our patient's psychotic relapses. This observation supports the view that CPK cannot be used to differentiate NMS from simple catatonia (Craddock et al, 1991). In our case, although neuroleptic medication was prescribed on both occasions, clinical symptoms of NMS were absent (e.g. fever, autonomic lability, muscle rigidity). The cause for the CPK rise remains speculative but in our patient we could not identify any other cause than the increase in muscular 'stress' associated with the catatonic symptoms.

More research is needed to identify a laboratory test which will help the early diagnosis of NMS in severely catatonic patients, and also to explore the meaning of raised CPK in catatonia. Do all patients with catatonia have high CPK or does it identify a population at risk for NMS?

Craddock, B., Craddock, N. \& Milner, G. (1991) CPK in NMS. British Journal of Psychiatry, 158, 130.

Fean, S. \& McGrath, M. G. (1990) Problems in diagnosing bipolar disorder in catatonic patients. Journal of Clinical Psychiatry, 51, 203-205.

RosebuSh, P. I. \& MAZUREK, M. F. (1991) Serum iron and neuroleptic malignant syndrome. Lancet, 338, 149-151.

White, D. A. C. \& Rosins, A. H. (1991) Catatonia: harbinger of the neuroleptic malignant syndrome. British Journal of Psychiatry. $158,419-421$.

\section{M.-A. DOMKEN} R. G. FARQUHARSON

\section{Hadrian Clinic}

Newcastle General Hospital

Westgate Road

Newcastle Upon Tyne

NE4 6BE

\section{A HUNDRED YEARS AGO}

\section{Paranoia and its relationships}

Dr. E. L. Dunn read a paper on paranoia, in which he remarked that chronic paranoia was generally admitted to occur in two forms: primary, which was the important and typical form, and secondary, which was the termination of a previous psychoneurotic state. Of the forms generally recognised, the most frequent was paranoia persecutoria; in it there were four stages: (1) restlessness and suspicion; (2) delusions of persecution, the delusional conceptions becoming systematised under the influence of aural and other hallucinations; (3) ideas of grandeur appeared; (4) dissolution of the delusional states and weak-mindedness. Dr. Dunn reviewed the literature of the subject at some length, and then proceeded to discuss the various symptoms seriatim. In referring to the statement of Metz and Roller that two cases had recovered after influenza, he remarked that remissions might occur with complete latency of symptoms. Dr. Dunn then discussed the various theories which had been advanced as to the pathological lesion underlying the condition. In secondary paranoia a small group of delusions remained and became systematised as an outcome of previous psycho-neurosis, almost always melancholia, profound weakening of all intellectual processes, monotonous delusions, and apathy were the characteristics of this condition, which ended in profound dementia. Having mentioned the occurrence of alcoholic paranoia, Dr. Dunn proceeded to discuss the diagnosis of the disorder.

\section{Reference}

British Medical Journal, 20 August, 1892, 411. 